\title{
Leveraging Materials in Topology Optimization: Inspiration from Design, Fashion, Art, and Architecture
}

\author{
NATASHA VERMAAK (1), ${ }^{1,4}$ JULIA DAVIY, ${ }^{2,5}$ \\ and VIRGINA SAN FRATELLO ${ }^{3,6}$ \\ 1.--Lehigh University, 19 Memorial Drive W, Bethlehem, PA 18015, USA. 2.-New Age \\ Lab, 1001 N. Federal Hwy, Hallandale Beach, FL 33009, USA. 3.-San Jose State \\ University, One Washington Square, San Jose, CA 95192, USA. 4.-e-mail: vermaak@lehigh.edu. \\ 5.—e-mail: hello@juliadaviy.com. 6.—e-mail: virginia.sanfratello@sjsu.edu
}

Topology optimization offers a mathematical framework to determine the most efficient material layout and shape for prescribed objectives, constraints, material behaviors, and operational conditions. While the application of topology optimization for continuum-scale structures and material behavior is quite mature, there remains great potential for topology optimization to advance the frontiers of materials design. This potential lies in thinking broadly about materials, waste, economy, craft, and design. Here inspiration is drawn from award-winning leaders and inventors in design, fashion, art, and architecture regarding the role of materials, as well as challenges and opportunities of designing with and for materials.

\section{INTRODUCTION}

In mathematics, topology is the study of properties of spaces that are invariant under any continuous deformation (that does not include tearing or gluing). The study of topology helps us to answer questions such as: How many holes are there in an object? What is the boundary of an object? Is a space connected? Topology optimization offers a mathematical framework to determine the most efficient material layout, connectivity, and shape for prescribed objectives, constraints, material behaviors, and operational conditions. ${ }^{1,2}$ There are many approaches for implementing topology optimization, but the following components are central to most formulations: definition of objective or cost functions, constraints dictated by physics or other considerations bounding the design variables, general physics or mathematical analysis tools for the problem of interest, optimization solution strategy or algorithm, and method for discretizing and describing an evolving topology.

While the application of topology optimization for continuum macroscale structures and material behaviors is quite mature, there remains great potential for topology optimization to advance the

(Received December 29, 2020; accepted April 30, 2021;

published online June 1, 2021) frontiers of materials design. For decades, topology optimization has been applied to continuum-scale structures such as aircraft and automotive components, buildings, and bridges. As reported in the 2019 National Academies Press Frontiers of Materials Research: A Decadal Survey, ${ }^{3}$ "topology optimization is pushing the frontiers of architected material design by decoupling and independently optimizing material properties and functionality."4,5 One opportunity lies in how materials are modeled and treated within optimization frameworks, including more understanding of materials and material behavior across the nano-, micro-, meso-, and macroscales. Some of the earliest examples of topology optimization (TO) for materials with tailored elastic properties ${ }^{6,7}$ perhaps required breakthroughs in additive manufacturing technologies before they were widely appreciated. More recently, exploiting understanding at the scale of material nano- and atomic structure has revealed vast opportunities for rational and optimal materials design, including atom-by-atom control. ${ }^{8}$ However, an important note here is that "material microstructure," rather than conjuring thoughts of scanning electron microscopy (SEM) images, is a term already used in TO communities, often referring to cellular architecture. Similarly, "material phase" is 
also a term used in TO that most often refers to a constituent material rather than a classical thermodynamic definition.

Nevertheless, beyond bridging ${ }^{9}$ or introducing ${ }^{8}$ material scales and identifying effective communication across disciplines, ${ }^{10}$ there is great potential in leveraging materials in topology optimization by thinking more broadly about materials, waste, economy, craft, and design. In the following, inspiration is drawn from award-winning leaders and inventors in design, fashion, art, and architecture regarding the role of materials, as well as challenges and opportunities in the design with/for/of materials. First, Julia Daviy, an inventor, environmentalist, and designer, gives an overview of how she understands the fusion of materials, sustainable fashion design, and the future of three-dimensional (3D) printing. Next, Virginia San Fratello, an architect whose whose research is at the convergence of digital, ecological, and building component design, ${ }^{11}$ gives her perspective on $3 \mathrm{D}$ printing of sustainable materials for the future. Finally, some concluding thoughts are presented.

The subsequent research articles give a brief snapshot of the state of the art in leveraging materials in topology optimization design frameworks. "Projection-Based Implicit Modeling Method (PIMM) for Functionally Graded Lattice Optimization" by H. Deng and A.C. To provides a densitybased TO framework for cellular materials and lattice structures that allows for flexibility in designing irregular and complex scaffolds. The results have biomimetic applications where material periodicity is not required and leads to extraordinary mechanical properties. "Additive Manufacturing of Topology Optimized Graded Porous Structures: An Experimental Study" by Z. Zhao and X.S. Zhang presents the utility of a multiporosity TO framework to optimize structures in the presence of material defects. This work in graded porous structures is at the intersection of designing for the absence of material and for imperfect materials. Finally, "Estimating Fluid PermeabilityPorosity Bounds with Topology Optimization" by L. Zhao, V.J. Challis, S. Ha, T.P. Weihs, and J.K. Guest (to appear in a separate $J O M$ issue), provides a unique application of TO to be the first to identify microstructure (or material architecture)-independent bounds between these two properties. This is a powerful example of leveraging TO to improve materials understanding. All of these studies are also intimately connected to the frontiers of additive manufacturing technologies.

\section{JULIA DAVIY: SUSTAINABLE FASHION DESIGN AND 3D PRINTING}

Where additive manufacturing and fashion meet, you will find Julia Daviy. As an emerging thought leader in the worlds of $3 \mathrm{D}$ printing, environmental science, and fashion design, Julia's award-winning approach to sustainable clothing and accessories has caught the attention of industry leaders around the globe, and her work continues to inspire others who share her vision of a zero-impact and positiveimpact society. As a co-founder of New Age Lab, Julia uses her extensive background in environmental science, clean-tech, and 3D printing to achieve significant reductions in $\mathrm{CO}_{2}$ emissions while creating sustainable fashion products.

Today, Julia Daviy is responsible for the full collection of clothing, accessories (bags), and jewelry (Figs. 1, 2 and 3). Daviy's products have been featured in New York Fashion Week 2018 and 2020, the Futurist Feminist Exhibition at the Boston Centre for the Arts, the Museum of Sustainability in Miami, the Rethinking Plastics Exhibition in The Netherlands, and other exhibitions. The professional accolades of Julia Daviy as a brand include the 2020 Edison Award (winner), the 2020 Fast Company Innovations by Design Awards (finalist), the 2020 Glossy Fashion Awards (silver), and the 2019 Eluxe Award (winner as an innovative sustainable brand). Daviy also holds a Guinness World Record for the largest 3D-printed flag. Before her foray into fashion, Julia spent a decade in the cleantech and renewable energy spaces.

Three-dimensional printing brings several advantages to the fashion industry from jewelry to shoes, bags, and clothes. Using digital design with further additive manufacturing, the $3 \mathrm{D}$ manufacture of products is practical not only at the prototype stage but increasingly for production. Opportunities created by 3D printing for consumer and fashion

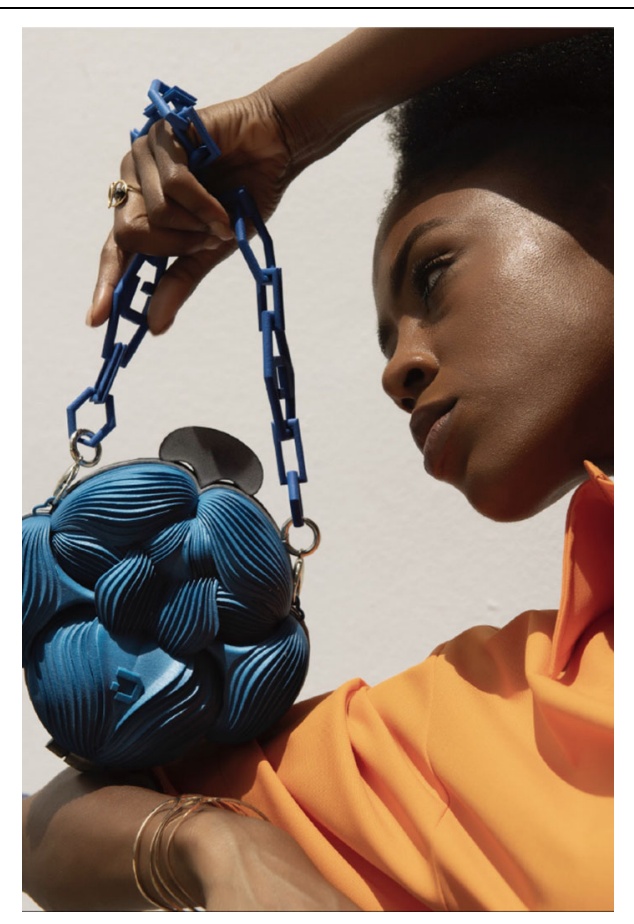

Fig. 1. Pandora bag from the Morphogenesis Collection (February 2020), SLS 3D printing, material: nylon. 


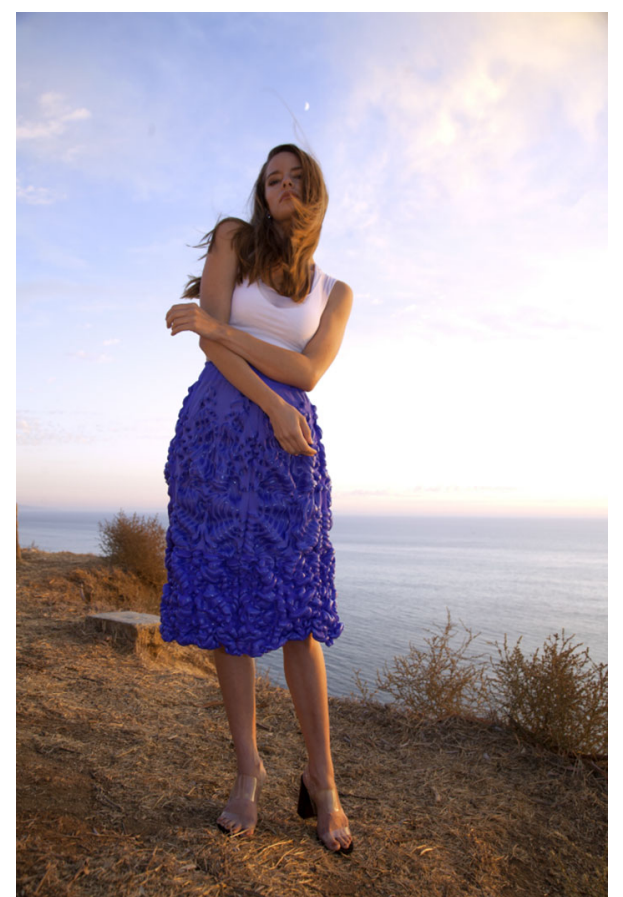

Fig. 2. The Blue Skirt (2019); 3D printing material: Thermoplastic elastomers (TPE) on recycled nylon fabric with changing of the shape.

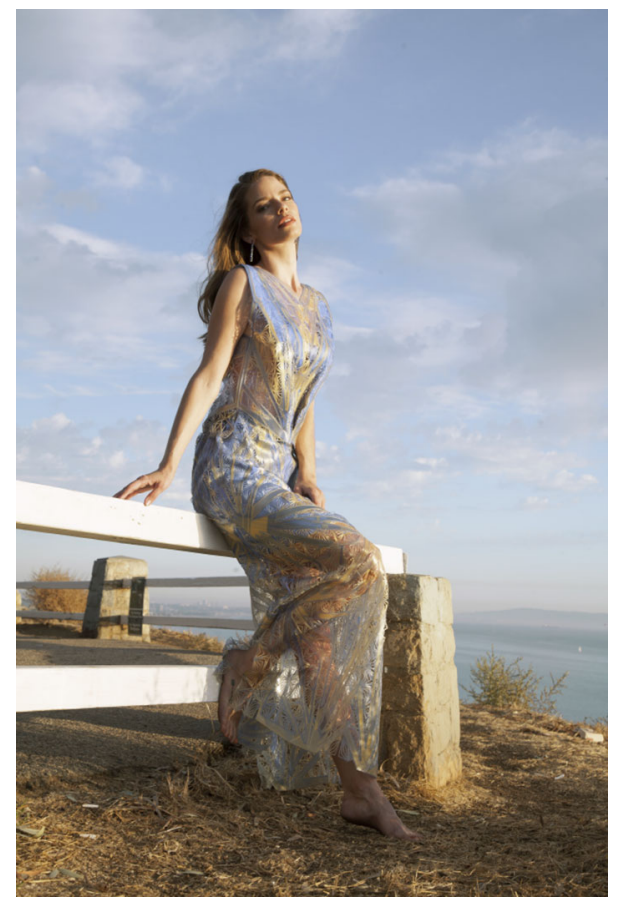

Fig. 3. The bicolored 3D-printed skirt and top (2019); FDM 3D printing, material: Thermoplastic elastomers (TPE).

products include: (a) Changing the overall manufacturing and business model. Designers and brands of any size can incorporate local $3 \mathrm{D}$ printing manufacturing into their business models and shorten their manufacturing and supply chains.
They can manufacture on demand and provide almost the same short delivery times as massmanufactured products. (b) A production model that is based on both digital design and digitized additive manufacturing and uses recyclable or biodegradable materials theoretically allows near-zero levels of waste, carbon emissions, and water usage. It makes radically more sustainable manufacturing possible. (c) Freedom of shape is becoming a growing trend in modern architecture and has had a significant influence on the fashion industry. Computational design and additive manufacturing make complex geometry possible. We are still in the early stages of exploring the potential applications of these tools. (d) Cost-effectiveness is the big promise of $3 \mathrm{D}$ printing. However, the cost of professional 3D printers and materials for 3D printing, which are traditionally more expensive than conventional manufacturing, is currently limiting this. (e) Ease of customization is a big benefit of $3 \mathrm{D}$ printing in general, and material science and engineering especially, in this field.

Opportunities for materials science relate to the development of "smart" textiles, which incorporate electronic capabilities into fabrics. Some types of 3D printing such as FDM and Polyjet allow 3D printing on fabrics as well as 3D printing using multimaterials. Another promising direction is the development of a range of materials that may change their properties under the influence of a changing physical and chemical environment. This, plus opportunities to create programmable textiles, could mean the creation of an entirely new generation of clothing, accessories, and jewelry, as well as have significant applications for home interior design. Imagine, for example, clothes that change their density, style, and color as a reaction to weather conditions.

Currently, the main limitation is the relatively small number of materials available for $3 \mathrm{D}$ printing. Also, there is a lack of 3D-printed materials with both high flexibility and durability that can compete with traditional materials such as leather in consumer goods production. Further, even though the market for "sustainable" materials is growing, the quality of these materials in many cases remains low and does not allow them to compete with conventional "non-sustainable" materials. Imagine the circular manufacturing model. At the heart of this, there is a completely recyclable material with the properties of modern nylon or cotton. In conventional manufacturing, this model will lead to measurable environmental and social impact. Alternatively, the implementation of digitalization and $3 \mathrm{D}$ printing can result in a zero or even positive environmental impact becoming a reality.

In the fashion industry, the challenge of producing $3 \mathrm{D}$ printing fabrics to replace traditional ones has not yet been achieved. An emerging alternative that is developing is $3 \mathrm{D}$ knitting, which is advanced knitting where it is possible to get manufactured 
ready-to-wear pieces of clothing from a digital design. The opportunity, and at the same time the challenge, for materials science here is the same as for the clothing industry in general, viz. with the creation of threads that have zero or positive impact on the environment.

\section{VIRGINIA SAN FRATELLO: EMERGING OBJECTS: 3D PRINTING SUSTAINABLE MATERIALS FOR THE FUTURE}

What if the world's waste materials could be transformed into sustainable building materials for the future? Can humble and traditional building materials, such as salt and mud, become sophisticated building materials for the twenty-first century? How can technologies such as additive manufacturing impact the way we attempt to solve social problems such as housing? These are all contemporary concerns that architecture must address if it is to stay relevant. Emerging Objects has attempted to answer some of these questions through the development of local, ubiquitous, and recycled materials for $3 \mathrm{D}$ printing. Chardonnay grape skins are an agricultural waste product that is left to rot in the field, in the Cabin of $3 D$-Printed Curiosities it is used as a local building material on the front façade in the form of planter tiles that hold succulents that thrive in the northern California environment (Fig. 4). While many of the proprietary powders and resins used in 3D printing are very expensive, the materials used for $3 \mathrm{D}$ printing described here, including soil and salt, are either free or very low cost, which makes 3D printing more accessible. The Saltygloo is printed using local salt harvested from the San Francisco Bay (Fig. 5), and the Casa Covida project uses locally sourced wild soil and clay from the jobsite, eliminating the need to purchase and ship materials around the world (Fig. 6).

At Emerging Objects, materials are developed for $3 \mathrm{D}$ printing and are transformed into blocks, tiles, and puddled coils to be used as building components that are easily handled and aggregable to create larger structures. Developing one's own materials, and using simple assembly methods for 3D-printed parts, opens the door for new material compositions within geometrically complex forms. It also reduces cost, and allows for new color variations and textures. The use of local, indigenous, and recycled materials in additive manufacturing not only addresses issues around the future of sustainability and economics but also creates new, contemporary craft and architectural traditions.

These materials and the architectural applications they are used in all speak to the possibility of a future that is just emerging, a future that takes advantage of powders that come from dust, waste, and traditional materials, on their journey to becoming part of a twenty-first-century architectural terroir that influences the meaningful crafting

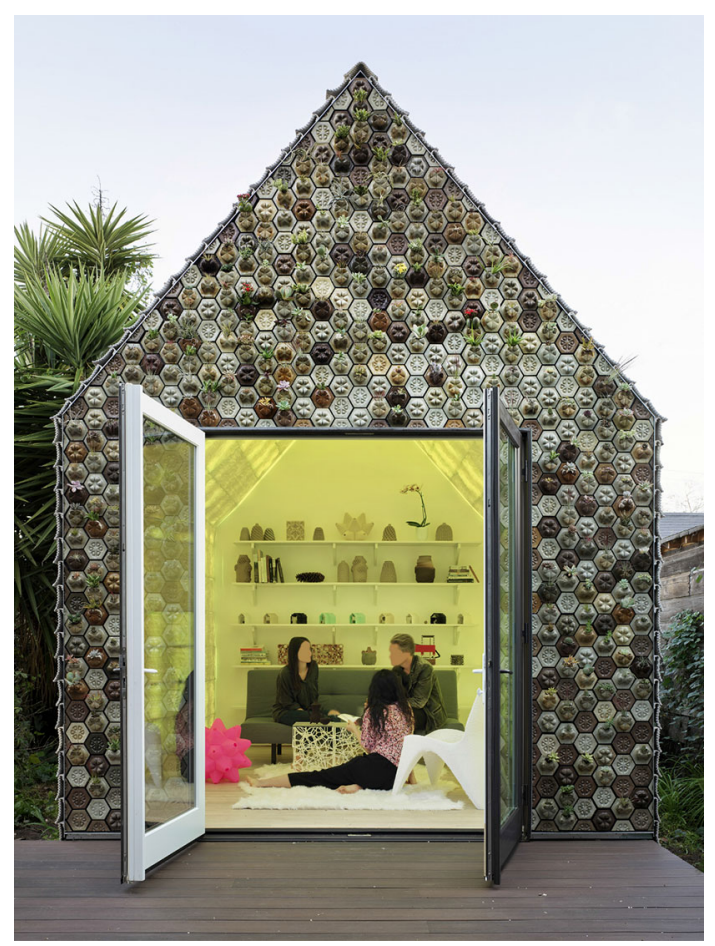

Fig. 4. The Cabin of 3D-Printed Curiosities: 3D-printed chardonnay, sawdust, and cement planter tiles.

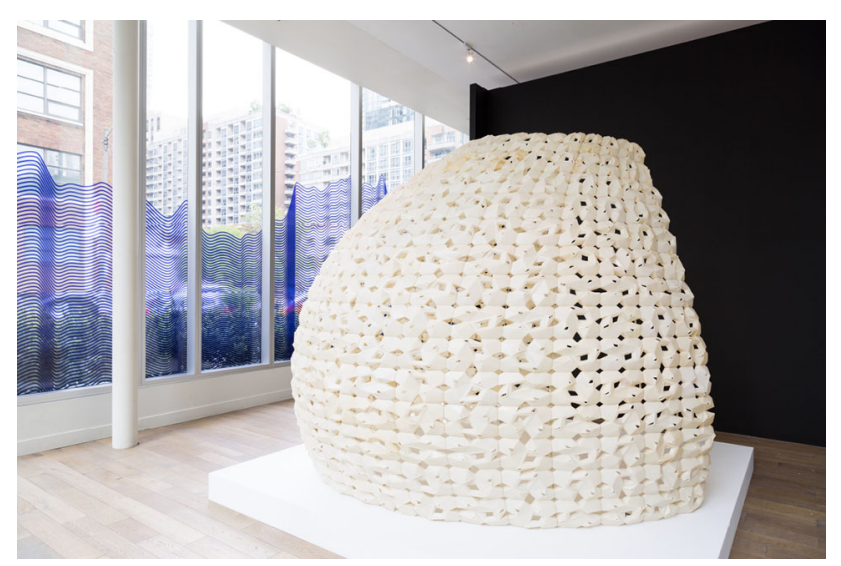

Fig. 5. Saltygloo exterior: 3D-printed salt.

of objects and buildings. As pointed out by Gareth Williams, "In order to retain relevance in the modern world, craft must engage with contemporary concerns. One of the most pressing issues today is the impact of production, consumption and disposal of goods upon the earth's resources and ecological balance". ${ }^{12}$ Three-dimensional printing also raises questions about its role in craft and how it might make bespoke architecture more accessible, as the objects and buildings produced are not necessarily handmade but are customized. However the close connections between design, iteration, technique, material behavior, analysis, and 


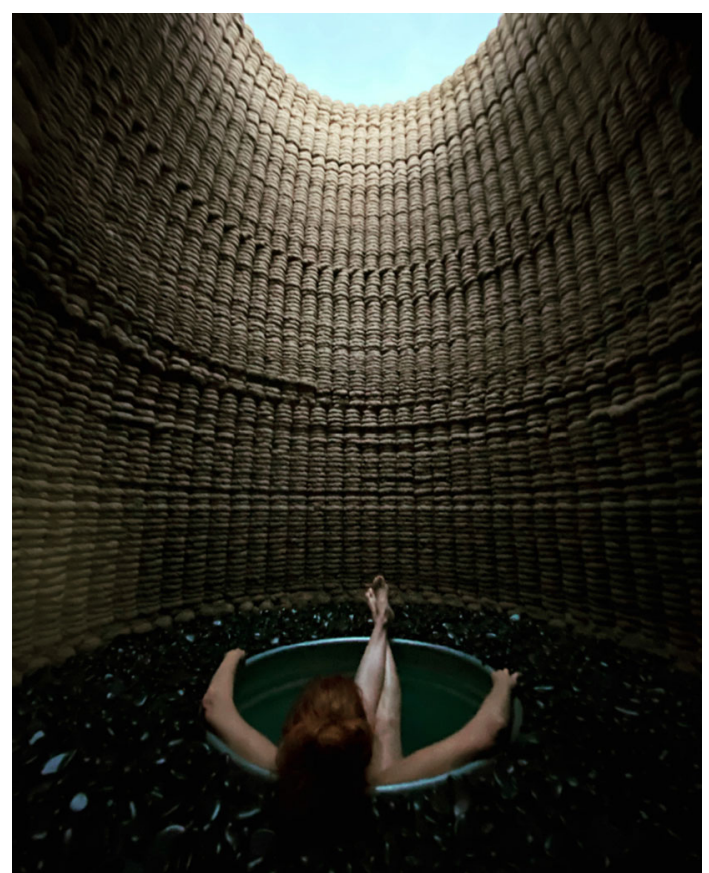

Fig. 6. Casa Covida: 3D-printed mud interior.

manufacturing suggest that 3D printing, especially when coupled with modes of production that employ materials from sustainable resources and waste streams, is becoming a contemporary form of manufacturing with increasing relevance.

\section{CONCLUDING REMARKS}

As emphasized by Julia Daviy and Virginia San Fratello, 3D printing is central to leveraging materials in modern design. With advances in process modeling, being able to better encode manufacturing rules in design algorithms to ensure realizable optimal designs is one research thrust. There are also many models, concepts, and tools from the materials science and engineering community, across the length and time scales critical to material behavior and performance, that have yet to be fully embraced. ${ }^{13}$ These include first-principles approaches, thermodynamic databases, and models for kinematically dominated mechanisms. From another perspective, questions such as where to put which knots are inspired by new models in the fundamental mechanics of textile science and engineering. ${ }^{14}$ When we ask a broader audience of designers what they consider as materials objectives and constraints, and which materials they find most challenging to work with and why, new research questions that may require reimagining topology optimization frameworks themselves may emerge.

All articles published under the topic "Leveraging Materials in Topology Optimization" in the July 2021 issue (vol. 73, no. 7) of JOM can be accessed at: http://link.springer.com/journal/11837/73/7/page/1.

\section{ACKNOWLEDGEMENTS}

N. Vermaak would like to acknowledge support from the US National Science Foundation (NSF) under Grant \#1825437. V. San Fratello would like to acknowledge the following for her projects: Cabin of 3D Printed Curiosities. Project team: Ronald Rael, Virginia San Fratello, Logman Arja, Hannah Cao, Sandy Curth, Barrak Darweesh, Yonghwan Kim, Daniel Komen, Cooper Rodgers, Alex Schofield, Phirak Suon, and Kent Wilson. Photography: Special thanks to Ehren Tool, Danny Defelici, Leonard Dodd at Autodesk, and The Bakar Fellows Program and Departments of Architecture and Art Practice at The University of California Berkeley. Additional thanks to Alisa Nadolishny, Natalie Yu, Anthony Gianini, and Sarah Rippee. Saltygloo. Project team: Ronald Rael, Virginia San Fratello, Seong Koo Le, and Eleftheria Stavridi. Photography courtesy of: The Design Exchange Toronto, CA. Special thanks to Professor Mark Ganter, Solheim Lab, University of Washington, Ehren Tool, Department of Art Practice, University of California Berkeley, Department of Architecture, University of California Berkeley, Department of Design, San Jose State University, Kwang Min Ryu, and Chaewoo Rhee. Casa Covida. Project team: Ronald Rael, Virginia San Fratello, Sandy Curth, Logman Arja, and Mattias Rael. Photograph courtesy of: Emerging Objects. Special thanks to Danny Defelici, Zaneta Defelici, and Dennis Vandergriff at 3D Potter, Darren Woo at the Woo Han Fai Group, Darell Chan at the Luk Hoi Tan Co., Ltd., Ivan Chen, Ehren Tool, The Bakar Fellows Program, The University of California Berkeley, and San Jose State University.

\section{CONFLICT OF INTEREST}

On behalf of all authors, the corresponding author states that there is no conflict of interest.

\section{REFERENCES}

1. M.P. Bendsoe and O. Sigmund, Topology Optimization: Theory, Methods, and Applications, 2nd edn. (Springer, Berlin, 2004).

2. G. Allaire, Shape Optimization by the Homogenization Method, vol. 146 (Springer, New York, 2002).

3. Engineering National Academies of Sciences and Medicine, Frontiers of Materials Research: A Decadal Survey (The National Academies Press, Washington, DC, 2019). https://d oi.org/10.17226/25244.

4. D. Pasini and J.K. Guest, MRS Bull. 44(10), 766 (2019).

5. M. Osanov and J.K. Guest, Annu. Rev. Mater. Res. 46, 211 (2016).

6. O. Sigmund, Design of material structures using topology optimization. Ph.D thesis.

7. O. Sigmund and S. Torquato, Appl. Phys. Lett. 69(21), 3203 (1996).

8. C.-T. Chen, D.C. Chrzan, and G.X. Gu, Nat. Commun. 11(1), 1 (2020).

9. R. Sivapuram, P.D. Dunning, and H. Alicia Kim, Struct. Multidiscip. Optim. 54(5), 1267 (2016).

10. The Minerals Metals \& Materials Society (TMS), Creating the Next-Generation Materials Genome Initiative Workforce 
(TMS, Pittsburgh, PA, 2019). https://doi.org/10.7449/mgiwo rkforce_1.

11. R. Rael and V.S. Fratello, Printing Architecture: Innovative Recipes for $3 D$ Printing (Princeton Architectural Press, Hudson, NY, 2018).

12. G. Williams, Creating lasting values, in The Persistence of Craft: The Applied Arts Today. ed. by P. Greenhalgh (Rutgers University Press, Piscataway, NJ, 2003), pp. 61-72.

13. N. Vermaak, G. Michailidis, A. Faure, G. Parry, R. Estevez, F. Jouve, G. Allaire, and Y. Bréchet, Topological optimiza- tion with interfaces, in Architectured Materials in Nature and Engineering (Springer, 2019), pp. 173-193.

14. V.P. Patil, J.D. Sandt, M. Kolle, and J. Dunkel, Science 367(6473), 71 (2020).

Publisher's Note Springer Nature remains neutral with regard to jurisdictional claims in published maps and institutional affiliations. 